\title{
Re-Plantation Revisited
}

\author{
MARIAM ISMAIL, M.D.; TAHER ISMAIL, M.D., F.R.C.S. and KARIMA ISMAIL, M.D \\ The Department of Plastic and Reconstructive Surgery, Faculty of Medicine, Cairo University
}

\begin{abstract}
Introduction: Replantation describes the re-attachment of a completely amputated part by restoration of arterial inflow and venous outflow. On the other hand Revascularization describes restoration of arterial inflow or venous outflow or both, to an incompletely amputated part, no matter how small the point of attachment.
\end{abstract}

The Aim of this Study is to: Reemphasize the Indications of replantation, propose doubtful cases for replantation, expand the indication of replantation, and explore the role of super microsurgery in replantation.

Patients and Methods: This retrospective study involving 437 hand units that have been transplanted after severed from the body from year 1990 to 2013 .

The cases were assorted according to, gender, age, type of trauma, ischemia time, and site of injury.

In 23 year period 437 hand units were replanted, surgical follow-up varied from 6 months to maximum of 4 years, with average of 2 years. $86 \%$ success rate ( 375 hand units), hand unit replantation regaining both vascularity and function.

Conclusion: Replantation is a very meticulous as well as demanding surgical procedure that requires a highly skilled team. If Replantation done successfully patient will achieve both functional and cosmetic results and better chance in life.

Key Words: Re-plantation - Amputation - Hands.

\section{INTRODUCTION}

Replantation describes the re-attachment of a completely amputated part by restoration of arterial inflow and venous outflow. On the other hand Revascularization describes restoration of arterial inflow or venous outflow or both, to an incompletely amputated part, no matter how small the point of attachment [1].

Re-plantation of a severed hand unit is a challenging decisionto Plastic Surgeons; as it depends on the condition of the patient, amputated part as well as the presence of an experienced microsurgery team. Defining successful replantaion is by restoring both vascularity as well as function of traumatically amputated part [1,2].

\section{PATIENTS AND METHODS}

This retrospective study involving 437 hand units that have been transplanted after severed from the body from year 1990 to 2013.

The cases were assorted according to:

Gender: Males: 281 cases, females: 156 cases.

Age: <2 yrs: 73 hand units. 2-14 yrs: 96 hand units. 14-35 yrs: 195 hand units. $>35$ yrs: 73 hand units.

Type of injury: Sharp guillotine injury: 88 hand units, crushed injury: 279 hand units and avulsion injury: 70 hand units.

Ischemia time, Warm ischemia time: 1-3 hrs: 76 hand units. 3-5 hrs: 128 hand units. 5-6 hrs: 20 hand units. $>6$ hrs: All cases received after $6 \mathrm{hrs}$ were discarded. Cold ischemia time: 1-5 hrs: 69 hand units. 5-8 hrs: 121 hand units. 8-12 hrs: 16 hand units. $>12$ hrs: 5 hand units. $>12$ hrs: 2 hand units.

N.B.: 1 case was received after $30 \mathrm{hrs}$ and another one was received after $42 \mathrm{hrs}$ and both were successfully replanted.

Site of injury:

1- Fingers.

2- Hemi hand (9, 6 transverse or 3 vertical).

3- Full hand (7 cases).

4- Forearm (3 cases, more proximal amputation).

Patients with amputations involving the upper extremity are candidates for replantation. In this study our absolute indications included, children, thumb, multiple fingers, proximal hand amputations (included transverse and vertical hemi hand amputations), and forearm level amputation provided suitable warm ischemia time [3]. 

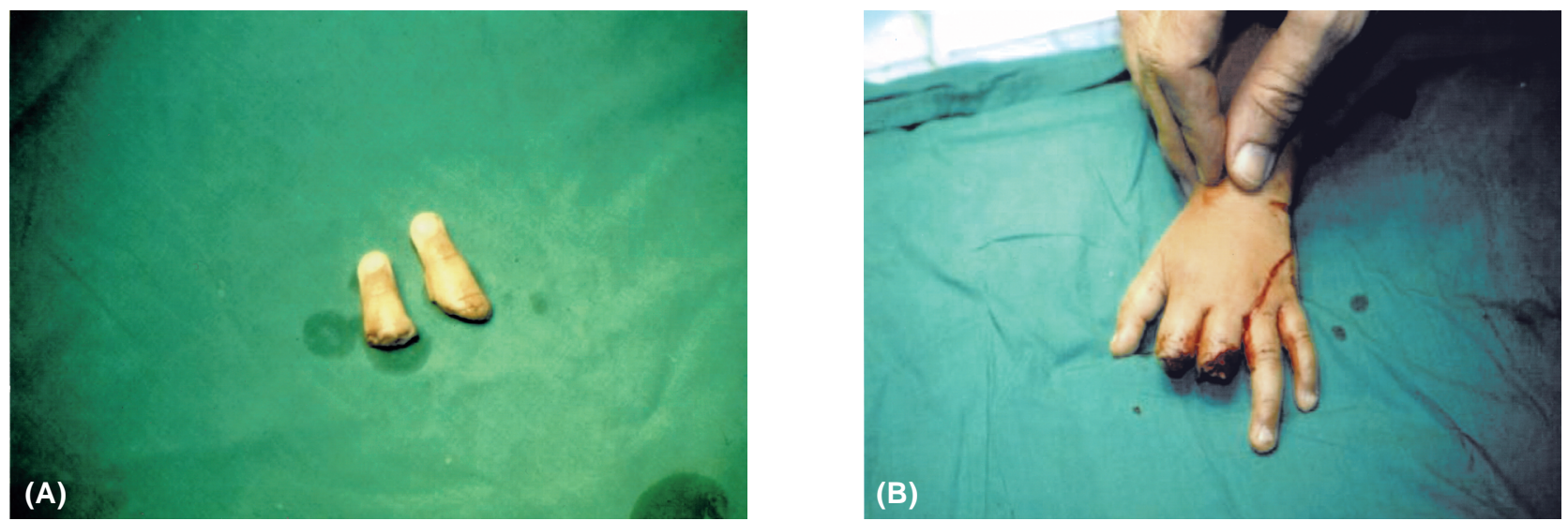

Fig. (1A,B): Multiple finger amputation in a child.

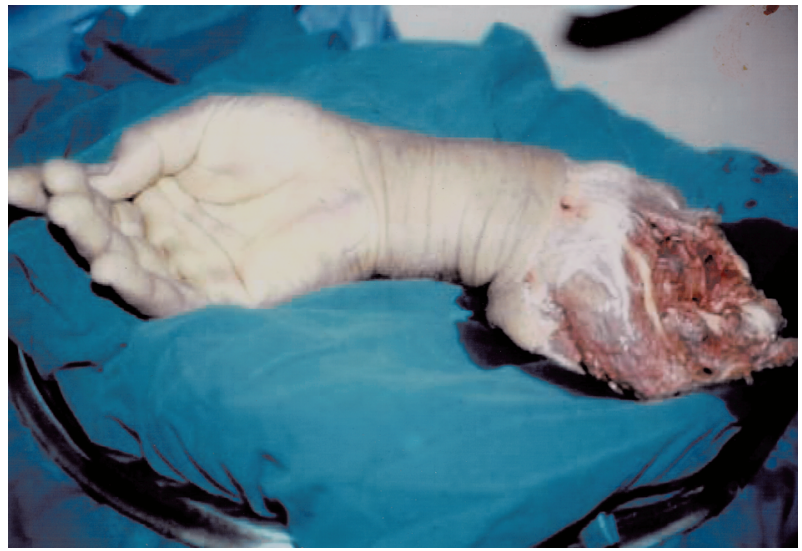

Fig. (1C): Mid forearm amputation.

Relative indications included single finger amputation (e.g.: index), multiple level amputations (taking in consideration patients' general condition), avulsion ring injuries and severely crushed injuries. In this study we included two more indications: (1) Multiple level injuries with omission of middle segment, (2) Super-microsurgery; (a) Children <2 yrs old i.e. vessels size smaller than $0.5 \mathrm{~mm}$. (b) Pieces of skin amputated with its artery and vein.

Once replantation decision is taken, the patient, amputated part and radiographs are taken to operating theater. While the patient is getting ready for anesthesia and surgery, the amputated part is thoroughly cleaned using dilutedbetadine solution together with antibiotic solution (combination of ciprofloxacin and garamycin) [4].

Meticulous debridement, identification and tagging of all structures (artery, vein; at least 2, nerves, flexor and extensor tendons) in the amputated part were done initially using loupe magnification followed by operating microscope.

The sequence of repair starts with bone shortening cut transversely using a power saw, in fingers

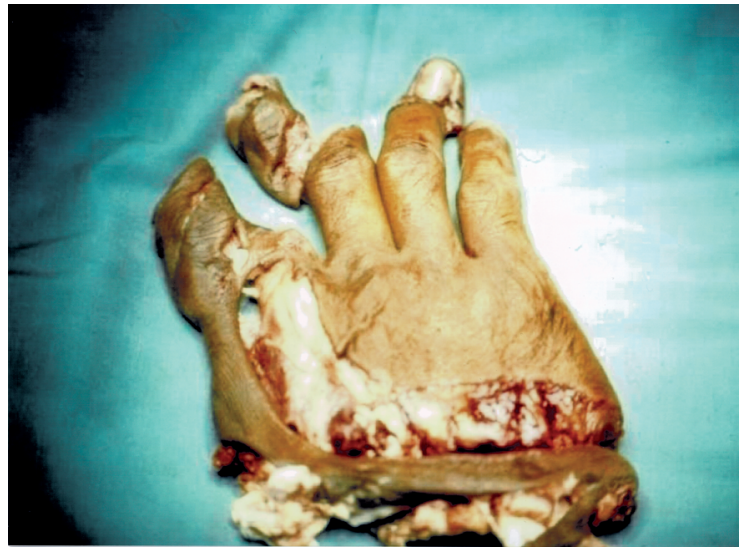

Fig. (1D): Mid palm amputation.

minimum of $0.2 \mathrm{~cm}$ to maximum $1.5 \mathrm{~cm}$ was done, proximal row carpectomy in hand amputation, and in cases of forearm amputation 2.5 to $4 \mathrm{~cm}$ shortening of radius and ulna, thus allowing primary nerve repair, end to end vascular anastomosis and tension free skin closure $[\mathbf{2 , 5 ]}$.

General anesthesia is used in all patients as well as padded tourniquet. The amputated stump thoroughly cleaned and prepped using betadine solution. Debridement, identification and tagging all structures (artery, vein; at least 2, nerves, flexor and extensor tendons) was done.

Rigid stable internal fixation is followed to ensure easy handling of vessels and nerves as well as allowing early protected motion of adjacent joints post operatively. Longitudinal wires were used in finger amputations, while dynamic compression plates were used in radius and ulna fixation [6].

After fixation repair of other structures takes the following sequence: Flexor tendons, extensor tendon, venous anastomosis, arterial anastomsis and finally nerve repair. 
In multiple finger amputations we started from middle to ring and lastly little. In cases involving thumb as well, we started with the thumb.

Tendons are repaired using $4 / 0$ prolene bunnel sutures as a core suture and 2-4 simple sutures 5/0 prolene suturing the paratenon.

Venous and arterial anastomoses were done using simple sutures starting with posterior wall followed by anterior wall. Prolene was used as suture material ranging from $7 / 0$ to $11 / 0$ prolene according to vessel size, site of injury and patient's age. Vein grafts were used in arterial repair of all avulsion injuries and in cases of severe intimal injury despite adequate bone shortening. Vein grafts are harvested from volar aspect of distal forearm, or veins from discarded amputated finger.

At least 2 veins repaired for every repaired artery to ensure adequate venous drainage thus avoiding post operative congestion and edema.

Epineural repair was done in nerve injuries using prolene as suture material ranging from 7/0$11 / 0$ depending on nerve size, site of injury, and patient's age.

Skin closure was done; in cases with tight closure split thickness graft is used. Latismus dorsi pedicled flap was used to cover anatomizes in forearm amputation.

Non-compressive bulky dressing was used together with hand elevation and special lamp directed to replanted part.

\section{Post operative care:}

Postoperative monitoring by well trained senior resident and nurse is mandatory in the first 24-48 hrs. Patient kept well hydrated, warm and pain free. Smoking is totally prohibited to avoid vasoconstrictive effect of nicotine, as well as caffeine. Antithrombotic medications were used, starting intra-operatively after release of vascular clamps $40 \mathrm{ml}$ of dextran 40 , as a bolus intravenously. Heparin is given as I.V. bolus $(5000 \mathrm{u})$ at time of revascularization and tourniquet release. Heparin dose is adjusted according partial thromboplastin time; it's used cautiously as it may cause hemorrhage thus jeopardizing the replant itself. Aspirin $80 \mathrm{mg}$ is given daily.

In case of venous congestion the exposed nail bed was scored with a needle and encouraged to bleed using heparin-soaked gauzes.

Revision procedures were done. Per-operative procedures such as repeating arterial or venous anastomosis during procedure were done. Other revision procedures in the form of tenolysis, and neurolysis were done 6 months to 1 year post operative.

\section{RESULTS}

In 23 year period 437 hand units were replanted, surgical follow-up varied from 6 months to maximum of 4 years, with average of 2 years. $86 \%$ success rate ( 375 hand units), hand unit replantation regaining both vascularity and function. 17\% (73 hand units) $<2$ yrs age, $21 \%$ (96 hand units) 2-14 yrs age. $44 \%$ of cases age ranged from 14-35 yrs and $16 \%$ cases above 35 yrs.

$20 \%$ presented with sharp guillotine injury, $64 \%$ presented with crush injury while $16 \%$ presented with avulsion injury.

Per-operative revision procedure i.e repeating anastomosis intra-operatively was done in $10 \%$ of hand units (43 hand units).

Re-exploration was done in first $24 \mathrm{hrs}$ in $3 \%$ of cases (13 hand units).

Vein grafts were used in $20 \%$ of hand units, in all avulsion injury units as well as indicated crushed injury units.

Primary skin closure was used in $97 \%$ of cases, $3 \%$ of cases required split thickness graft closure to avoid tight closure which might affect anastomosis. In one case pedicled latismus dorsi flap was used.

Since it's a retrospective study we didn't have enough data to evaluate each joint function. Our data collection regarding functional outcome depended on patients' records, photos and patients we were able to reach.

It is a subjective analysis depending on surgeon's opinion, pre-operative and post-operative photo records as well as patients' questionnaire. All data collected and classified to 3 grades, good, fair and poor regarding flexion, extension of finger/s, grip and pinch power restored.

All fingers whether single or multiple finger injuries distal to MP joint 67\% 293 hand unit showed good result regarding function.

All fingers amputated through MP joint particularly index suffered of stiffness poor function.

2 cases out of the $3 \mathrm{mid}$ forearm amputations showed good functional outcome after 4 yrs follow- 
up. The $3^{\text {rd }}$ case required tenolysis as patient was suffering from stiffness and repair of the ulnar nerve as it wasn't repaired during first setting.

Total number of full hand amputation included 7 cases, 4 out of the 7 cases scored good regarding aesthetic and functional outcome, 2 scored fair and 1 scored poor.

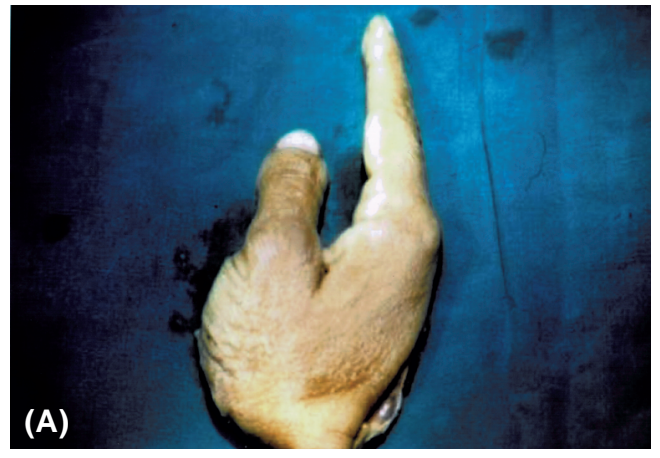

Fig. (2): RT. Radial hemi-hand amputation, (A and B) Pre- operative.
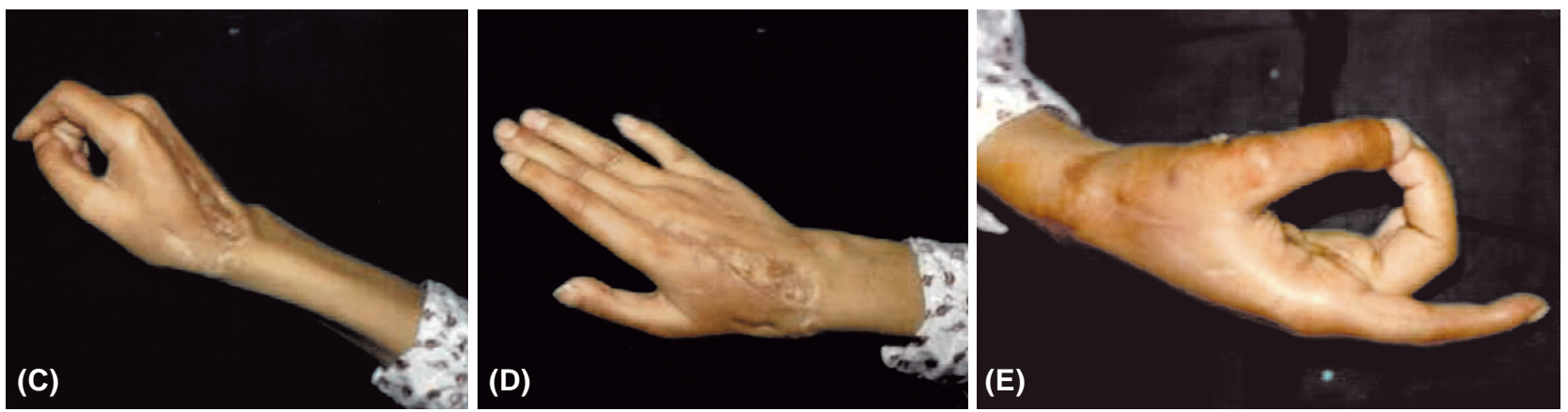

Fig. (2C,D,E): 1 year post-operative.
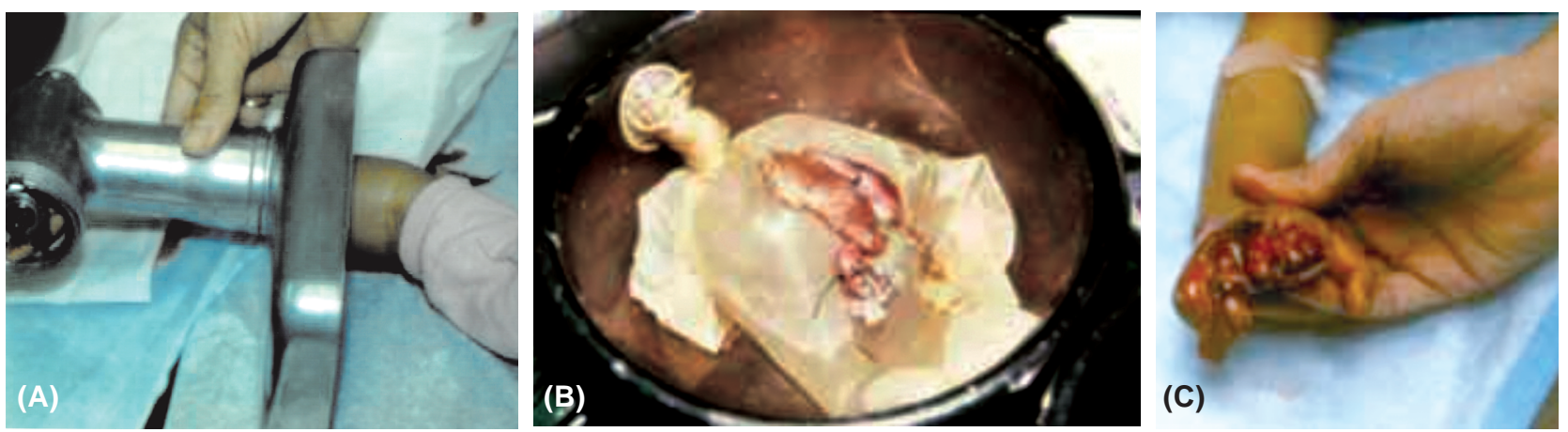

Fig. (3A,B,C): 2 yrs child with amputaed middle 3 fingers from meat grinder.
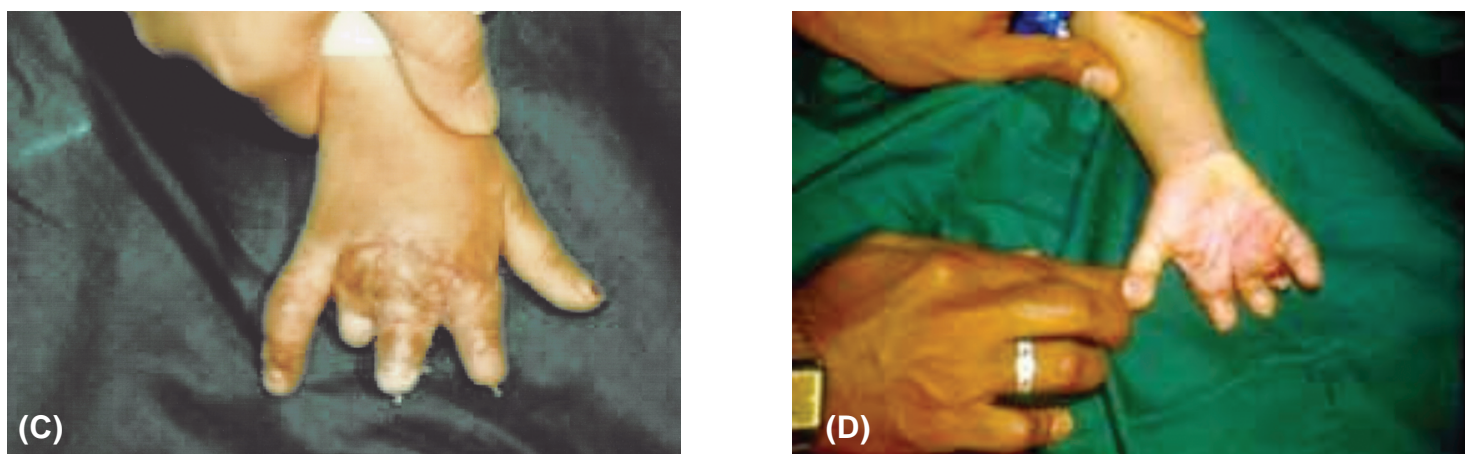

Fig. (3D,E): 4 months post-operative. 

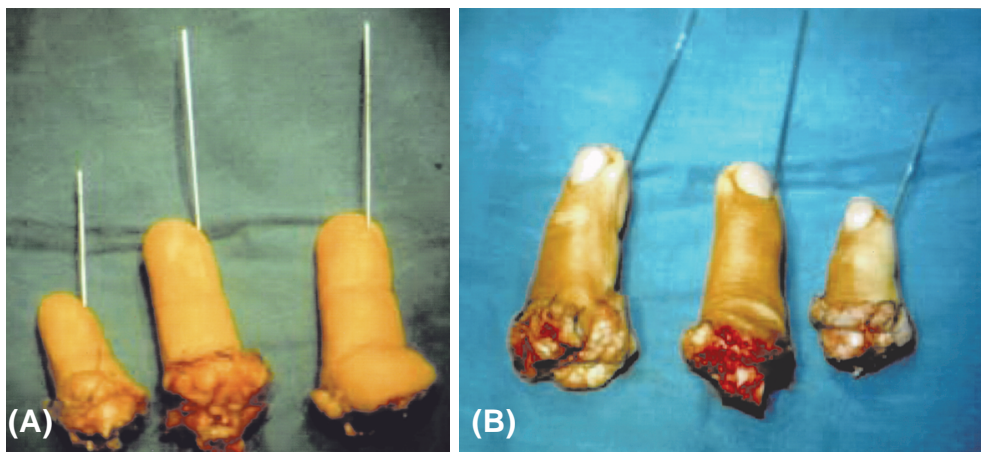

Fig. (4A,B): Ampuated medial 3 fingers.

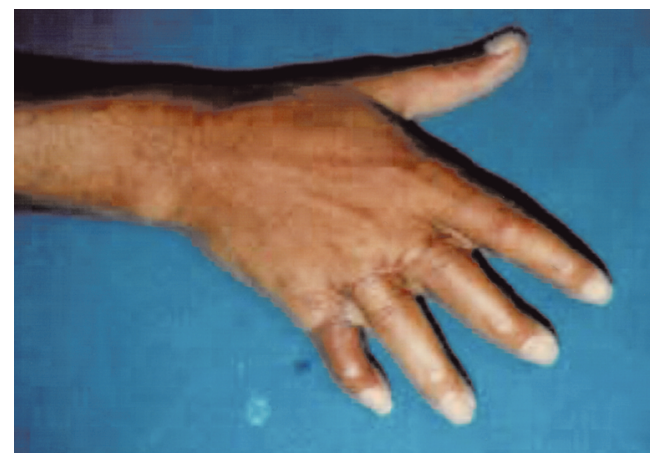

Fig. (4C): 6 months post operative.

\section{Complications:}

Early:

1- Loss of replanted hand unit. (whether arterial or venous occlusion).

\section{2- Hematoma.}

$14 \%$ failure rate which are followed by revision amputation procedures.

Per-operative revision procedure in the form of repeating arterial or venous anastomosis was done in 10\% (34 hand units).

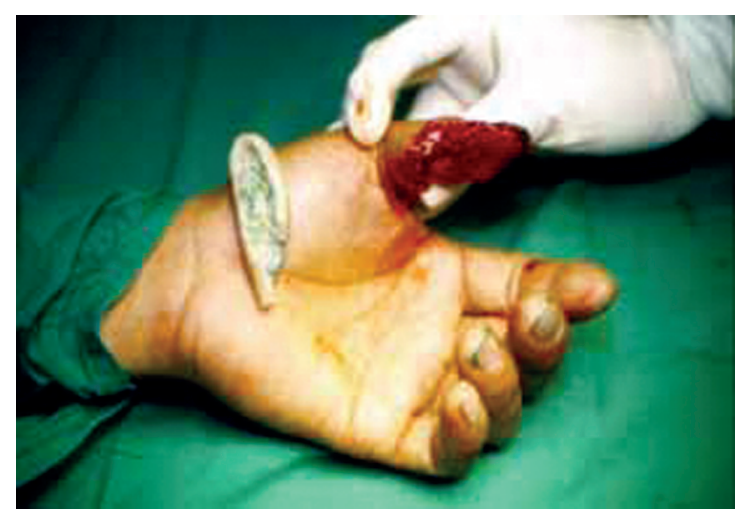

Fig. (5A): Amputated skin, volar aspect of left thumb.

\section{DISCUSSION}

Re-plantation of severed hand unit is a challenging decision that requires highly skilled surgeon as well as well trained team. Patients with severed hand unit deserve every chance to re-plant the amputated hand unit, provided that both vascularity and function is restored [7].

This retrospective study involved 437 hand units that were transplanted after severed from the body from year 1990 to 2013 with $86 \%$ success rate. The $14 \%$ failure rate was mainly in the early years due to lack of replantation tools, developing surgical skills, lack of well trained stuff. With time tools were more available together with increased
Late:

1- Malunion and nonunion.

2- Joint stiffness.

3- Tendon adhesions.

4- Muscle contracture.

5- Cold intolerance [8].

$12 \%$ of re-planted hand units required tenolysis procedure to relief stiffness, while approximately $15 \%$ of hand units needed post-operative neurolysis to relief pain.

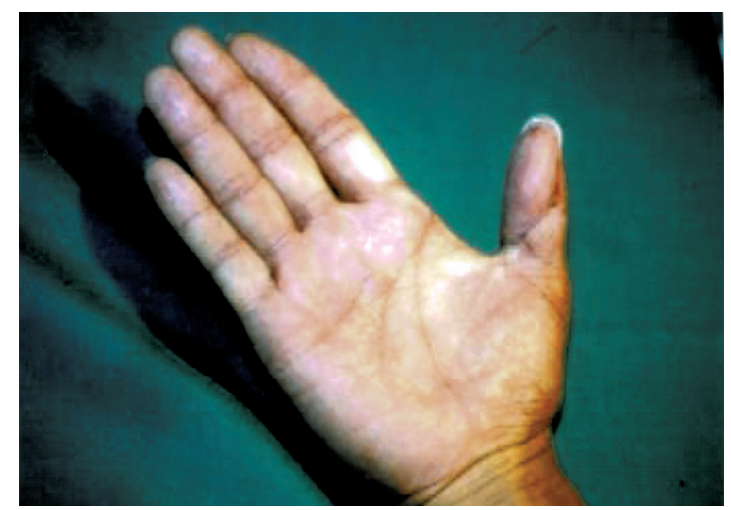

Fig. (5B): Re-plantation of amputated skin, super microsurgery.

experience of the trained microsurgery team. Severely crushed hand unit, old age, smokers, and lack of patient compliance also contributed to failure rate.

Younger patients showed better healing as they have a better chance of their nerves growing back and they regain more feeling and movement in the replanted part.

Regarding site of injury, better aesthetic and functional recovery were found in the more distally replanted parts. In cases with non-joint affection showed more movement and early return to function than those who have injured the joints. 
A cleanly severed part; guillotine injuries usually regained better function after replantation than crush or avulsion injury cases. $20 \%$ of cases needed vein grafts to bypass zone of injury, together with planning and debridement leading to increased operation time.

Hand units received with warm ischemia time more than 6 hours especially in more proximal amputation were discarded. Successful re-plantation of two hand units was done after 30 and 42 hours of cold ischemia time, so we strongly recommend proper preservation of amputee should be taught to all personals in emergency departments as they can change patients' life [7]. Primary tension free arterial, venous and nerve repair gives by far better guaranteed results then grafts usage. Achieving that is done by bone shortening with maximum of $1.5 \mathrm{~cm}$ in fingers and $4 \mathrm{~cm}$ in radius and ulna. The more the rigid the fixation the easier the manipulation and the better the bone union post-operative thus avoiding non and mal-union complications [9].

We strongly recommend for each arterial repair at least 2 veins repaired to avoid venous congestion thus avoiding arterial thrombosis and jeopardizing the anastomosis [10].

Repair of flexor tendons in zone 2 is always controversial, in our study we repaired only flexor digitorum profundus tendon as the only flexor tendon in zone 2 injuries and we encouraged early mobilization of joints that gave better functional results.

Tension free skin closure together with fasciotomy, drain application and post-operative hand elevation are strongly recommendedand mandatory as they alleviates the effect of edema or hematoma that may jeopardize the anastomsis [11].

In cases with multiple level amputations we recommend an omission or discarding part of hand unit in order to give a chance for patient to have ordinary life. Even small pieces of skin with their arterial supply could be transplanted.

\section{Conclusion:}

Replantation is a very meticulous as well as demanding surgical procedure that requires a highly skilled team. Fortunately upper extremity amputations are the commonest and luckly the distal amputations are the frequent and with better results. Every patient presents in emergency department with severed hand unit deserve the right and every chance for replantation. If Replantation successfully done patient will achieve bothfunctional and cosmetic results and better chance in life.

\section{REFERENCES}

1- Buntic R.F. and Brooks D.: Standardized protocol for artery-only fingertip replantation. J. Hand Surg. Am. Sep., 35 (9): 1491-6, 2010.

2- Herrera F.A., Lee C.K., Brooks D., Buntic R. and Buncke G.M.: Simultaneous double second toe transplantation for reconstruction of multiple digit loss in traumatic hand injuries. J Reconstr. Microsurg. Jul., 25 (6): 369-76, 2009.

3- Buntic R.F., Brooks D. and Buncke G.M.: Index finger salvage with replantation and revascularization: Revisiting conventional wisdom. Microsurgery, 28 (8): 612-6, 2008.

4- Brooks D., Buntic R.F. and Taylor C.: Use of the venous flap for salvage of difficult ring avulsion injuries. Microsurgery, 28 (6): 397-402, 2008.

5- Brooks D., Buntic R.F., Kind G.M., Schott K., Buncke G.M. and Buncke H.J.: Ring avulsion: Injury pattern, treatment, and outcome. Clin. Plast. Surg. Apr., 34 (2): 187-95, viii, 2007.

6- Colwell A.S., Buntic R.F., Brooks D., Wright L., Buncke G.M. and Buncke H.J.: Detection of perfusion disturbances in digit replantation using near-infrared spectroscopy and serial quantitative fluoroscopy. J. Hand Surg. Am. Mar., 31 (3): 456-62, 2006.

7- Buncke G.M., Buntic R.F. and Romeo O.: Pediatric mutilating hand injuries. Hand Clin. Feb., 19 (1): 121-31, 2003.

8- Brooks D., Buntic R. and Buncke H.J.: Use of a venous flap from an amputated part for salvage of an upper extremity injury. Ann. Plast. Surg. Feb., 48 (2): 189-92, 2002.

9- Agarwal J.P., Trovato M.J., Agarwal S., Hopkins P.N., Brooks D. and Buncke G.: Selected outcomes of thumb replantation after isolated thumb amputation injury. $\mathrm{J}$. Hand Surg. Am. Sep., 35 (9): 1485-90, 2010.

10- Chao J., English J.M. and Tittle B.: Microsurgery: Free tissue transfer and replantation. Selected Readings in Plastic Surgery, Dallas, Texas, 2000.

11- Goel A., Navato-Dehning C., Varghese G. and Hassanein K.: Replantation and amputation of digits: User analysis. Am. J. Phys. Med. Rehabil, 74: 134-138, 1995. 